\title{
The Determinants of Caregiver use and its Costs for Elderly Inpatients in Korea: A study applying Andersen's behavioral model of health care utilization and replacement cost method
}

\section{Jennifer Ivy Kim}

The Catholic University of Korea

Sukil Kim ( $\square$ sikimmd@gmail.com )

The Catholic University of Korea

\section{Research Article}

Keywords: Health expenditure, Elderly patient, Caregiving, Informal caregiver

Posted Date: April 20th, 2021

DOl: https://doi.org/10.21203/rs.3.rs-417694/v1

License: (c) (i) This work is licensed under a Creative Commons Attribution 4.0 International License. Read Full License 


\section{Abstract}

Objectives: The average annual healthcare expenditure among elderly patients in Korea is increasing rapidly in indirect healthcare sectors, requiring an understanding of factors related to the use of both formal and informal caregivers. This study aims to analyze the factors related to caregiver use and caregiving costs among hospitalized patients due to acute illness or exacerbation of chronic diseases and whether they differ by age group.

Methods: A total of 1,550 study participants from the Korea Health Panel Study Data 2017, consisting of 819 elderly inpatients and 731 nonelderly inpatients, were used for the analysis. Costing methods were applied according to the major type of caregiver during hospitalization. Predisposing, enabling, and need factors were considered to determine the possible factors associated with caregiver use. A two-part model was applied to analyze the factors related to care receiving and estimate incremental costs from care after stratifying subjects by age.

Results: Elderly inpatients who used tertiary hospitals (OR: 2.44 , p-value $<0.00$ ), received financial support (OR: $2.70, p$-value $<0.00$ ), and had disability status (OR: 1.76 , p-value $<0.05)$ were more likely to rely on a caregiver, while elderly inpatients living by themselves had a lower probability of using caregivers (OR: 0.48 , $p$-value $<0.00$ ). Also, there was a positive association between caregiving costs and the number of chronic diseases among elderly inpatients $(\beta: 0.66, p$-value $<0.05)$ with incremental costs of $\$ 567$.

Conclusion: This study presented significant health-related and socio-environmental characteristics to the use of formal and informal caregiving and its related expenditures. Therefore, healthcare management plans that encompass clinical and social levels should be implemented to ease the caregiver burden.

Trial registration: Retrospectively registered

\section{Background}

Statistics Korea and the National Health Insurance Review and Assessment report that the share of elderly individuals in South Korea over 65 years of age was 15.7\% in 2020 (1), with average annual healthcare expenses for an elderly patient of US\$ 4,147 (a currency exchange rate of Korean Won \#109,950 to US dollar $\$ 100$, the same below), which is expected to rise shortly (2). Healthcare expenditures from not only medical services but also caregiving have resulted in significant burdensome in households with elderly patients in Korea. According to Korean Social Trends 2019 reports, the average time spent on family caregiving was 29.5 hours per week, with a maximum caregiving time of 168 hours per week, implying that the social costs derived from informal care should be considered when evaluating healthcare burdens (3). Along with universal healthcare coverage for the Korean population with a singlepayer scheme and mandatory enrollment of all citizens, the Korean government has aimed to support inpatients in receiving both nursing and caregiving services through a workforce consisting of a registered nurse and assistant personnel in designated hospitals with a Comprehensive Nursing Service (CNS) (4). However, some studies have shown that nurses working in CNS wards experience greater job 
distress and higher turnover rates than those working in the general unit (5), which may degrade not only hospital operations but also increase the unmet needs of elderly patients with severe symptoms due to the imbalance in medical service supply and demand (4).

Healthcare service use and healthcare cost payments between the elderly and nonelderly adults are distinctive. Elderly patients who are more exposed to major chronic diseases (6) and experience limited improvement from treatment, such as major abdominal surgery (7), are usually in demand of assist their daily functioning and resilience to cope with stressors from the treatment process. Nonelderly patients have diverse health conditions and various socioeconomic features to those 65 years and older (8), which implies that it is difficult to definitively assess the risk factors related to healthcare cost burden among relatively younger patients. Some international studies have demonstrated the possibility of indirect healthcare costs placing an economic burden on elderly patients. In the case of Tomita's study, indirect healthcare costs constituted the majority of total healthcare expenditures among homecare service receivers, and those who require intensive care experience a financial burden due to both direct and indirect healthcare costs in Japan (9). In Italy, caregivers supporting patients with advanced ovarian cancer experienced caregiving time cost burdens not only from spending time assisting patients but also from reducing their work hours (10). Also, Joo reported considerable informal caregiving costs per stroke survivor in United States and identified the need to study potential factors affecting caregiving burdens on the family (11). However, little is known about the factors associated with the use of informal caregivers and their social costs in Korea. Several studies have focused on direct healthcare expenditures or formal caregiving in Korea have been done such as, governmental benefits for long-term institutional formal care lowered medical expenses but had a limited effect on reducing informal care (12). Additionally, long-term patients' gender, age, and health status are related to whether they hire paid caregivers $(13,14)$, whereas determinants of the use of both formal and informal caregivers have not yet been discussed.

Therefore, this study aimed to analyze the socioeconomic factors affecting caregiving costs among hospitalized patients due to acute illness or exacerbation of chronic diseases and determine whether they differ by age group. The specific aims are to determine the factors associated with using both formal and informal caregivers, identify the factors affecting both formal and informal caregiving costs, and compare those factors by age group.

\section{Methods}

\section{Data source}

This study uses the Korea Health Panel Study Data (KHP) in 2017 for the analysis. The KHP provides information about individual healthcare behavior, health status, usage of health services, and healthcare expenditures and is jointly carried out by the Korea Institute for Health and Social Affairs and the National Health Insurance Service (15)

\section{Study participants}


A total of 2,145 individuals who had experienced hospitalization within a year were selected from the data. Those who had no caregiving records $(n=30)$ or hospital admission cost records $(n=286)$ were excluded. Of those who had fully answered the survey items, inpatient service users younger than 20 years old $(n=205)$ and those who had been admitted to the hospital for childbirth $(n=72)$ or for cosmetic surgery $(n=2)$ were also excluded. Therefore, a total of 1,550 study participants, consisting of 819 elderly patients aged more than 65 years and 731 nonelderly patients, were included in the analysis (Fig. 1).

\section{Definition of Variables}

Formal and informal caregivers. Caregiving is defined as assisting acutely hospitalized patients' daily activities, engaged by paid caregivers, voluntary organizations, or relatives. The types of caregivers were classified according to their relationship with the patients. Formal caregivers were defined as those who were financially paid for their caregiving services, whereas informal caregivers were relatives or unpaid nonrelatives of the inpatients. Informal caregiving at the patient's residence after discharge from the hospital was excluded from the cost estimation to maintain consistency as caregiving was limited to nursing at the time of hospitalization.

Caregiving cost estimation. The study participants answered questions about their major caregivers during hospitalization. Formal caregiving costs were calculated by multiplying the average formal caregiver payment per day by the total number of days that caregiving services were received. The replacement cost method was used to estimate informal caregiving costs, based on the assumption that hiring paid caregivers can be a substitute for engaging in informal caregiving activities $(11,15)$. The reason for using the replacement cost method is that the care costs are limited to those incurred while caring for patients using healthcare services, and informal care provided during hospitalization is a suitable substitute for paid caregivers, from which follows the relevance of measuring the economic value of informal caregiving (16). Additionally, there are limitations in applying different costing measurements such as the opportunity cost method because most informal care providers, even if they are not engaged in economic activities, do not provide detailed information that can be used to distinguish and estimate the value of the time given up to provide actual care. Paid care services are readily apparent alternatives to the informal care provided by medical institutions that occur during treatment, such as inpatient care, therefore, the replacement cost method is a more reasonable approach than the opportunity cost method, which requires estimating caregivers' wage levels $(15,16)$. The costs of informal caregivers' time were estimated by applying the market price of equivalent services. The average per-day payment to paid caregivers used to estimate the cost of daily informal caregiving is based on the Healthcare Experience Survey 2018 (17). Therefore, informal caregiving costs were estimated by multiplying the total number of days of hospitalized by the daily cost of informal caregiving.

\section{Independent variables}

The sociodemographic characteristics of individuals from different contextual backgrounds influence healthcare service use (18). Predisposing, enabling, and need factors, as defined by Andersen's behavior model, are variables that can appropriately explore the size of the association between the use of 
caregiving (19-22). This study includes gender, age, educational level, and household type as predisposing factors. Enabling factors consisted of the household income level, which was measured by dividing annual income by the square root of the household size and was then divided into quintiles. Also, the types of health insurance that inpatients held at the time of hospitalization were classified into two types: national health insurance $(\mathrm{NHI})$ or the medical aid program, supporting low-income households, or patients with rare, intractable chronic disease. However, this study excluded the enrollment status of Long-term Care Insurance (LTCI), which covers the direct healthcare expenses of elderly patients over 65 years old who obtained prior approval for services through medical assessment of their functional ability, because fewer than $1 \%$ of participants were enrolled in this scheme. Therefore, it was concluded that LTCI would have a mere impact on the analysis. Additionally, CNS could not be accounted for because information related to CNS began to be collected only in 2018. Instead, survey results regarding the receipt of financial aid for hospital admission from nongovernmental sources such as nonprofit organizations or private insurance were used to measure the receipt of support for the hospital admission. Finally, the medical institutions in which patients had been majorly hospitalized were categorized into two groups: general hospitals with a minimum of 100 beds providing specialist services in major medical fields and local hospitals with at least 30 beds. In case of the need factors, the number of chronic diseases, the Charlson comorbidity index, and having a physical or psychological disability, as determined by codes converted from patients' reports of receiving a medical diagnosis, were used to assess the need for healthcare services among inpatients. The Korean Standard Classification of Disease version 7 (KCD-7) is the Korean modification of the International Statistical Classification of Diseases and Related Health Problems (ICD-10) for international comparisons. In this study, the types of diseases are defined by using categories at the block level $(23,24)$. The types of disease with which patients were diagnosed are classified into eight categories: cancer, diseases of the eye and adnexa, diseases of the circulatory system, diseases of the respiratory system, diseases of the digestive system, diseases of the musculoskeletal system, and connective tissues, injury, poisoning and certain other consequences of external causes, and other symptoms were considered. Additionally, the severity level of the disease was assessed using the Charlson comorbidity index (CCl) score, which calculated as in a previous study using the appropriately aligned KCD-7 codes (25). Finally, survey results regarding having received physical or psychological disabilities from the medical institution were accounted to include patients' endogenous health issues that require essential assistance.

\section{Statistical Analysis}

Caregiving costs means the sum of payments to paid caregivers and the estimated informal caregiving costs. The distribution of caregiving costs is composed of zero for non-users and positive values for those receiving caregiving services. Therefore, a two-part model was used to determine the factors related to using assistance from caregivers and estimating incremental caregiving costs after stratifying subjects according to their age. In the first part, the probability of receiving caregiving was estimated using logistic regression analysis. Among those who were assisted by caregivers during hospitalization, the factors that determine caregiving costs were analyzed in the second part using a generalized linear model (GLM) with a log link and gamma distribution. GLM was used to analyze the two-part model and 
identify the incremental effects of caregiving costs. The variance inflation factors confirmed that there were no multicollinearity problems, and the model specification test showed that there were no misspecification errors. All estimates were weighted to represent the general population. Stata (Stata Corp, Texas, US) version 16 was used for all statistical analyses.

\section{Results}

\section{General characteristics of elderly and nonelderly inpatients}

Table 1 demonstrates the sociodemographic characteristics of the study participants. Elderly patients made up $36.0 \%$ of the study participants, and most of them were female $(58.7 \%)$, with an average age of $75.5 \pm 7.3$ years. The majority of these patients were supported by caregivers during hospitalization (69.1\%) and answered that hospital charges for inpatient services were burdensome (79.4\%), while more than half had received inpatient services at a tertiary hospital (63.0\%). Additionally, these patients were generally residing with their spouse (47.0\%) and had the lowest household income (40.0\%). $17.7 \%$ of them had more than two chronic diseases and $19.6 \%$ of them had a CCl score of at least 1 . Nonelderly patients accounted for $64.0 \%$ of the study patients, and more than half of them were female with an average age of $48.3 \pm 11.9$ years. Most had used tertiary hospitals $(54.0 \%)$ and experienced burdens in paying hospital services (74.0\%). Most nonelderly patients were living with their spouse and other family members $(70.9 \%)$ and had the highest household incomes (30.5\%). In terms of health conditions, $16.3 \%$ of them had more than 2 chronic diseases, while $11.2 \%$ had $\mathrm{CCl}$ scores greater than 1 .

Table 1. General Characteristics of elderly and nonelderly inpatients 


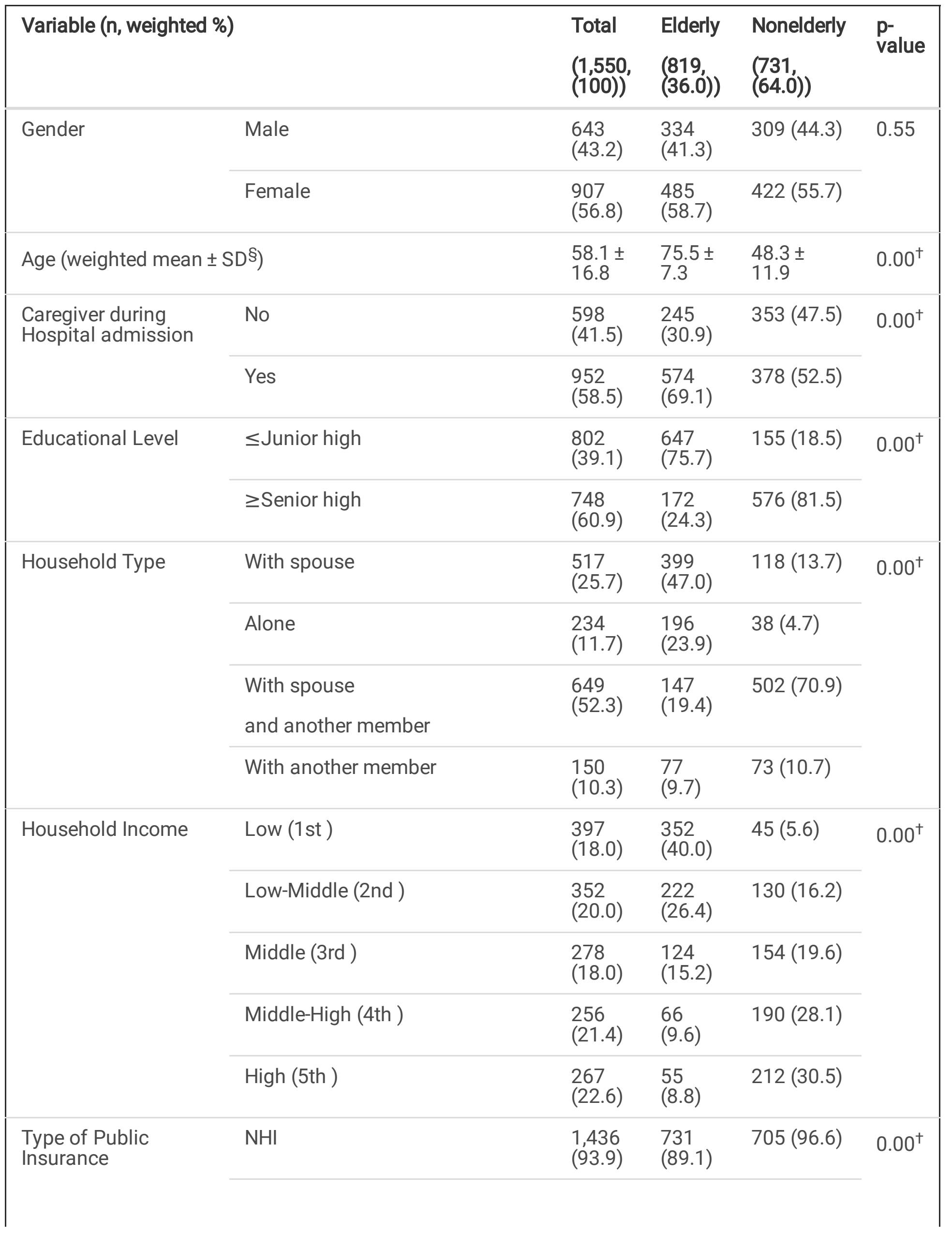




\begin{tabular}{|c|c|c|c|c|c|}
\hline \multicolumn{2}{|l|}{ Variable (n, weighted \%) } & \multirow{2}{*}{$\begin{array}{l}\text { Total } \\
(1,550, \\
(100))\end{array}$} & \multirow{2}{*}{$\begin{array}{l}\text { Elderly } \\
\left(\begin{array}{l}819 \\
(36.0))\end{array}\right. \\
88 \\
(10.9)\end{array}$} & \multirow{2}{*}{$\begin{array}{l}\text { Nonelderly } \\
(731, \\
(64.0))\end{array}$} & \multirow{2}{*}{$\begin{array}{l}\mathrm{p}- \\
\text { value }\end{array}$} \\
\hline & Medicaid & & & & \\
\hline \multirow[t]{2}{*}{ Financial Support } & No & $\begin{array}{l}704 \\
(43.4)\end{array}$ & $\begin{array}{l}396 \\
(48.1)\end{array}$ & $308(40.7)$ & \multirow[t]{2}{*}{$0.01^{*}$} \\
\hline & Yes & $\begin{array}{l}846 \\
(56.6)\end{array}$ & $\begin{array}{l}423 \\
(51.9)\end{array}$ & $423(59.3)$ & \\
\hline \multirow[t]{2}{*}{$\begin{array}{l}\text { Financial Burden due } \\
\text { to Hospital Charges }\end{array}$} & $\begin{array}{l}\text { Not burdensome at all } \\
\text { Somewhat manageable }\end{array}$ & $\begin{array}{l}352 \\
(24.0)\end{array}$ & $\begin{array}{l}161 \\
(20.6)\end{array}$ & $191(26.0)$ & \multirow[t]{2}{*}{$0.00^{+}$} \\
\hline & $\begin{array}{l}\text { Somewhat burdensome } \sim \text { Very } \\
\text { burdensome }\end{array}$ & $\begin{array}{l}1,198 \\
(76.0)\end{array}$ & $\begin{array}{l}658 \\
(79.4)\end{array}$ & $540(74.0)$ & \\
\hline \multirow[t]{2}{*}{$\begin{array}{l}\text { Types of Medical } \\
\text { Institution }\end{array}$} & General hospital & $\begin{array}{l}656 \\
(42.7)\end{array}$ & $\begin{array}{l}311 \\
(37.0)\end{array}$ & $345(46.0)$ & \multirow[t]{2}{*}{$0.00^{+}$} \\
\hline & Tertiary hospital & $\begin{array}{l}894 \\
(52.3)\end{array}$ & $\begin{array}{l}508 \\
(63.0)\end{array}$ & $386(54.0)$ & \\
\hline \multirow[t]{2}{*}{$\begin{array}{l}\text { Number of Chronic } \\
\text { diseases }\end{array}$} & 1 & $\begin{array}{l}1,301 \\
(83.2)\end{array}$ & $\begin{array}{l}680 \\
(82.3)\end{array}$ & $621(83.7)$ & \multirow[t]{2}{*}{0.30} \\
\hline & $\geq 2$ & $\begin{array}{l}249 \\
(16.7)\end{array}$ & $\begin{array}{l}139 \\
(17.7)\end{array}$ & $110(16.3)$ & \\
\hline \multirow[t]{2}{*}{$\mathrm{CCl}$} & 0 & $\begin{array}{l}1,303 \\
(85.8)\end{array}$ & $\begin{array}{l}661 \\
(80.4)\end{array}$ & $642(88.8)$ & \multirow[t]{2}{*}{$0.00^{+}$} \\
\hline & $\geq 1$ & $\begin{array}{l}247 \\
(14.2)\end{array}$ & $\begin{array}{l}158 \\
(19.6)\end{array}$ & $89(11.2)$ & \\
\hline \multirow{6}{*}{$\begin{array}{l}\text { Type of Diagnosed } \\
\text { Disease at the Time } \\
\text { of Hospital } \\
\text { Admission }\end{array}$} & Cancer (Neoplasms) & $\begin{array}{l}193 \\
(13.0)\end{array}$ & $\begin{array}{l}91 \\
(11.7)\end{array}$ & $102(13.6)$ & \multirow[t]{6}{*}{$0.00^{+}$} \\
\hline & Diseases of the eye and adnexa & $\begin{array}{l}143 \\
(7.5)\end{array}$ & $\begin{array}{l}104 \\
(12.6)\end{array}$ & $39(4.7)$ & \\
\hline & $\begin{array}{l}\text { Diseases of the circulatory } \\
\text { system }\end{array}$ & $\begin{array}{l}141 \\
(8.7)\end{array}$ & $\begin{array}{l}95 \\
(12.6)\end{array}$ & $46(6.6)$ & \\
\hline & $\begin{array}{l}\text { Diseases of the respiratory } \\
\text { system }\end{array}$ & $\begin{array}{l}114 \\
(7.3)\end{array}$ & $\begin{array}{l}66 \\
(7.8)\end{array}$ & $48(7.0)$ & \\
\hline & Diseases of the digestive system & $\begin{array}{l}151 \\
(10.8)\end{array}$ & $\begin{array}{l}69 \\
(8.7)\end{array}$ & $82(11.9)$ & \\
\hline & $\begin{array}{l}\text { Diseases of the musculoskeletal } \\
\text { system and connective tissue }\end{array}$ & $\begin{array}{l}292 \\
(20.2)\end{array}$ & $\begin{array}{l}134 \\
(16.3)\end{array}$ & $158(22.4)$ & \\
\hline
\end{tabular}




\begin{tabular}{|c|c|c|c|c|c|}
\hline \multicolumn{2}{|c|}{ Variable (n, weighted \%) } & $\begin{array}{l}\text { Total } \\
(1,550, \\
(100))\end{array}$ & $\begin{array}{l}\text { Elderly } \\
\left(\begin{array}{l}819, \\
(36.0)\end{array}\right)\end{array}$ & $\begin{array}{l}\text { Nonelderly } \\
(731, \\
(64.0))\end{array}$ & \multirow{2}{*}{$\begin{array}{l}\mathrm{p}- \\
\text { value }\end{array}$} \\
\hline & $\begin{array}{l}\text { Injury, poisoning and certain } \\
\text { other consequences of external } \\
\text { causes }\end{array}$ & $\begin{array}{l}235 \\
(14.5)\end{array}$ & $\begin{array}{l}119 \\
(14.1)\end{array}$ & $116(14.7)$ & \\
\hline & Other symptoms and signs & $\begin{array}{l}281 \\
(18.0)\end{array}$ & $\begin{array}{l}141 \\
(16.2)\end{array}$ & $140(19.1)$ & \\
\hline \multirow{2}{*}{$\begin{array}{l}\text { Physical } \\
\text { /Psychological } \\
\text { Disability }\end{array}$} & No & $\begin{array}{l}1,362 \\
(89.5)\end{array}$ & $\begin{array}{l}673 \\
(82.2)\end{array}$ & $689(93.7)$ & \multirow[t]{2}{*}{$0.00^{\dagger}$} \\
\hline & Yes & $\begin{array}{l}188 \\
(10.5)\end{array}$ & $\begin{array}{l}146 \\
(17.8)\end{array}$ & $42(6.3)$ & \\
\hline
\end{tabular}

Note

$\S \mathrm{SD}=$ Standard Deviation ${ }^{*} \mathrm{p}<0.05+\mathrm{p}<0.00$

\section{Average Total Healthcare Expenditures for Hospital Admission}

The total healthcare costs of hospital admissions include hospital charges for inpatient services and caregiving costs. Hospital charges for inpatient services are divided into three categories: covered by $\mathrm{NHI}$, paid through cost-sharing, and paid out of pocket. The charges in each category were calculated according to the study participants' age group and experience of receiving care (Table 2). Elderly patients with caregivers paid US $\$ 3,966 \pm 5,158$ for their hospital admissions, while nonelderly patients supported by caregivers spent US $\$ 2,906 \pm 2,916$. Elderly patients' average caregiving cost due to hiring formal caregivers was US\$1,291 $\pm 1,436$ and the estimated average cost of informal caregiving was US\$1,034 $\pm 2,283$. However, elderly patients without caregivers had lower total healthcare costs (US\$ $1,359 \pm 1,605$ ) than the nonelderly patients (US\$1,796 $\pm 2,012$ ). In case of nonelderly patients, those who were cared for by formal caregivers paid US\$ $846 \pm 229$, while the average cost for informal caregiving was estimated to be US $\$ 592 \pm 830$. After summing both hospital charges for inpatient services and caregiving costs, elderly patients with caregivers were found to have had greater total health care costs (US\$ $5,016 \pm 6,625$ ) than the nonelderly patients (US\$3,501 $\pm 3,357$ ). Therefore, elderly and nonelderly patients spent $21 \%$ and $17 \%$ of their healthcare expenditure on caregiving costs, respectively (Fig. 2). 
Table 2

Average Total Healthcare Expenditures during Hospital Admission

\begin{tabular}{|c|c|c|c|c|c|c|}
\hline \multirow{2}{*}{$\begin{array}{l}\text { Expenditure } \\
\text { Category } \\
\text { (Weighted Mean } \pm \\
\text { SD§ (US\$)) }\end{array}$} & \multicolumn{3}{|c|}{ Elderly Inpatients } & \multicolumn{3}{|c|}{ Nonelderly Inpatients } \\
\hline & $\begin{array}{l}\text { With } \\
\text { Caregiver } \\
(n=574)\end{array}$ & $\begin{array}{l}\text { Without } \\
\text { Caregiver } \\
(n=245)\end{array}$ & $\begin{array}{l}\text { Total } \\
(n= \\
819)\end{array}$ & $\begin{array}{l}\text { With } \\
\text { Caregiver } \\
(n=378)\end{array}$ & $\begin{array}{l}\text { Without } \\
\text { Caregiver } \\
(n=353)\end{array}$ & $\begin{array}{l}\text { Total } \\
(n=731)\end{array}$ \\
\hline $\begin{array}{l}\text { A. Hospital Charges } \\
\text { for Inpatient } \\
\text { Services }\end{array}$ & $\begin{array}{l}3,966 \pm \\
5,158\end{array}$ & $\begin{array}{l}1,358 \pm \\
1,605\end{array}$ & $\begin{array}{l}3,156 \pm \\
4,535\end{array}$ & $\begin{array}{l}2,906 \pm \\
2,916\end{array}$ & $\begin{array}{l}1,796 \pm \\
2,012\end{array}$ & $\begin{array}{l}2,378 \pm \\
258\end{array}$ \\
\hline (a) Covered by $\mathrm{NHI}$ & $\begin{array}{l}2,715 \pm \\
3,938\end{array}$ & $919 \pm 1,227$ & $\begin{array}{l}2,157 \pm \\
3,440\end{array}$ & $\begin{array}{l}1,688 \pm \\
2,068\end{array}$ & $\begin{array}{l}1,031 \pm \\
1,474\end{array}$ & $\begin{array}{l}1,376 \pm \\
20,016\end{array}$ \\
\hline $\begin{array}{l}\text { (b) Paid through } \\
\text { cost sharing }\end{array}$ & $\begin{array}{l}575 \pm \\
791\end{array}$ & $234 \pm 343$ & $\begin{array}{l}469 \pm \\
702\end{array}$ & $\begin{array}{l}453 \pm \\
527\end{array}$ & $257 \pm 315$ & $\begin{array}{l}360 \pm \\
450\end{array}$ \\
\hline $\begin{array}{l}\text { (c) Paid out of } \\
\text { Pocket }\end{array}$ & $\begin{array}{l}675 \pm \\
1,242\end{array}$ & $204 \pm 369$ & $\begin{array}{l}529 \pm \\
1,074\end{array}$ & $\begin{array}{l}763 \pm \\
1,160\end{array}$ & $507 \pm 775$ & $\begin{array}{l}641 \pm \\
1,003\end{array}$ \\
\hline B. Caregiving Costs & $\begin{array}{l}1,053 \pm \\
2,233\end{array}$ & - & $\begin{array}{l}1,053 \pm \\
2,233\end{array}$ & $\begin{array}{l}596 \pm \\
825\end{array}$ & - & $\begin{array}{l}596 \pm \\
825\end{array}$ \\
\hline $\begin{array}{l}\text { (d) Formal } \\
\text { Caregiving Costs }\end{array}$ & $\begin{array}{l}1,291 \pm \\
1,436\end{array}$ & - & $\begin{array}{l}1,291 \pm \\
1,435\end{array}$ & $\begin{array}{l}846 \pm \\
229\end{array}$ & - & $\begin{array}{l}846 \pm \\
229\end{array}$ \\
\hline $\begin{array}{l}\text { (e) Informal } \\
\text { Caregiving Costs }\end{array}$ & $\begin{array}{l}1,034 \pm \\
2,283\end{array}$ & - & $\begin{array}{l}1,034 \pm \\
2,284\end{array}$ & $\begin{array}{l}592 \pm \\
830\end{array}$ & - & $\begin{array}{l}592 \pm \\
830\end{array}$ \\
\hline $\begin{array}{l}\text { Total Healthcare } \\
\text { Cost }(A+B)\end{array}$ & $\begin{array}{l}5,016 \pm \\
6,625\end{array}$ & $\begin{array}{l}1,359 \pm \\
1,605\end{array}$ & $\begin{array}{l}3,884 \pm \\
5,826\end{array}$ & $\begin{array}{l}3,501 \pm \\
3,357\end{array}$ & $\begin{array}{l}1,796 \pm \\
2,012\end{array}$ & $\begin{array}{l}2,691 \pm \\
2,924\end{array}$ \\
\hline
\end{tabular}

\section{Determinants of caregiver use and caregiving costs among elderly and nonelderly inpatients}

The two-part model estimated for the total study participants illustrates that elderly inpatients were more likely to receive support from caregivers (OR: $1.86, p<0.00)$ and spent $37 \%$ more on caregiving costs ( $\beta$ : $0.37, p<0.00$ ) than nonelderly inpatients (Table 3 ). In the case of household type, inpatients living alone (OR: $0.40, p<0.00$ ) or those who were residing with their spouse and other family members (OR: $0.64, p<$ $0.05)$ were less likely to use caregivers during hospitalization. However, whether inpatients lived alone had no significant relationship with caregiving expenditures $(\beta: 0.06, p=0.70)$. On the other hand, households comprising spouses and other family members had a significant tendency to spend $31 \%$ more on caregiving costs than households with spouses only $(\beta: 0.31, p<0.05)$. For household income status, inpatients from a low-to middle-income household was less likely to use a caregiver than 
inpatients from the lowest income group (OR: $0.53, p<0.00)$, but there was no significant relationship with such inpatients' caregiving expenditures $(\beta: 0.05, p=0.73)$. Those who received financial support for their healthcare expenditure both used caregivers more (OR: $1.36, p<0.05)$ and spent $40 \%$ of more on caregiving costs $(\beta: 0.40, p<0.00)$ than non- receivers, and both estimates are statistically significant. Also, inpatients diagnosed with more than two chronic diseases had $46 \%$ higher caregiving costs than inpatients with a single disease $(\beta: 0.46, p<0.00)$. Similarly, inpatients with a CCl score greater than 1 had significantly higher caregiving costs: they spent $33 \%$ more on caregiving costs $(\beta: 0.33, p<0.05)$ than inpatients with a $\mathrm{CCl}$ score of zero. Inpatients admitted to a tertiary medical institute were more likely to be cared for by caregivers (OR: $1.92, p<0.00)$ than general hospital users. 
Table 3

Analysis of caregiver use and its costs among elderly and nonelderly inpatients

\begin{tabular}{|c|c|c|c|}
\hline \multirow[t]{2}{*}{ Variable } & & $\begin{array}{l}\text { Logit Model }(n= \\
1,550)\end{array}$ & $\begin{array}{l}\text { GLM Model }(n= \\
\text { 952) }\end{array}$ \\
\hline & & $\begin{array}{l}\text { Odds Ratio (95\% } \\
\mathrm{CI} \&)\end{array}$ & $\begin{array}{l}\text { Coefficient }(95 \% \\
\mathrm{CI} \S)\end{array}$ \\
\hline $\begin{array}{l}\text { Gender } \\
\text { (Ref: Male) }\end{array}$ & Female & $0.84(0.64 \sim 1.12)$ & $\begin{array}{l}-0.09(-0.28 \sim \\
0.10)\end{array}$ \\
\hline $\begin{array}{l}\text { Age Group } \\
\text { (Ref: Nonelderly group) }\end{array}$ & Elderly group & $1.86^{\dagger}(1.33 \sim 2.58)$ & $\begin{array}{l}0.37^{\dagger} \\
0.65)\end{array}(0.10 \sim$ \\
\hline $\begin{array}{l}\text { Educational Level } \\
\text { (Ref: } \geq \text { Senior High) }\end{array}$ & $\leq$ Junior High & $1.07(0.77 \sim 1.50)$ & $\begin{array}{l}0.27^{*}(0.16 \sim \\
0.54)\end{array}$ \\
\hline \multirow{3}{*}{$\begin{array}{l}\text { Household Type } \\
\text { (Ref: With spouse) }\end{array}$} & Alone & $0.40^{\dagger}(0.26 \sim 0.61)$ & $\begin{array}{l}0.06(-0.26 \sim \\
0.38)\end{array}$ \\
\hline & $\begin{array}{l}\text { With spouse and another } \\
\text { member }\end{array}$ & $0.64^{*}(0.45 \sim 0.91)$ & $\begin{array}{l}0.31^{*} \\
0.58)\end{array}(0.04 \sim$ \\
\hline & With another member & $0.72(0.45 \sim 1.17$ & $\begin{array}{l}0.22(-0.09 \sim \\
0.54)\end{array}$ \\
\hline \multirow{4}{*}{$\begin{array}{l}\text { Household Income } \\
\text { (Ref: Low (1st)) }\end{array}$} & Low-Middle (2nd ) & $0.53^{\dagger}(0.36 \sim 0.79)$ & $\begin{array}{l}0.05(-0.24 \sim \\
0.35)\end{array}$ \\
\hline & Middle (3rd ) & $0.93(0.58 \sim 1.50$ & $\begin{array}{l}-0.08(-0.41 \sim \\
0.25)\end{array}$ \\
\hline & Middle-High (4th ) & $0.90(0.54 \sim 1.48)$ & $\begin{array}{l}-0.14(-0.49 \sim \\
0.20)\end{array}$ \\
\hline & High (5th ) & $0.93(0.57 \sim 1.54)$ & $\begin{array}{l}-0.06(-0.51 \sim \\
0.37)\end{array}$ \\
\hline $\begin{array}{l}\text { Type of Public Insurance } \\
\text { (Ref: NHI) }\end{array}$ & Medicaid Type 1/Type 2 & $0.79(0.48 \sim 1.30)$ & $\begin{array}{l}0.34(-0.03 \sim \\
0.72)\end{array}$ \\
\hline $\begin{array}{l}\text { Financial Support } \\
\text { (Ref: Non-Receiver) }\end{array}$ & Receiver & $1.36^{*}(1.04 \sim 1.77)$ & $\begin{array}{l}0.40^{\dagger}(0.23 \sim \\
0.58)\end{array}$ \\
\hline $\begin{array}{l}\text { Number of Chronic } \\
\text { diseases } \\
\text { (Ref: 1) }\end{array}$ & $\geq 2$ & $0.79(0.56 \sim 1.11)$ & $\begin{array}{l}0.46^{*}(0.10 \sim \\
0.83)\end{array}$ \\
\hline $\begin{array}{l}\text { CCI } \\
\text { (Ref: 0) }\end{array}$ & $\geq 1$ & $1.31(0.88 \sim 1.94)$ & $\begin{array}{l}0.33^{*}(0.01 \sim \\
0.66)\end{array}$ \\
\hline
\end{tabular}




\begin{tabular}{|c|c|c|c|}
\hline \multirow[t]{2}{*}{ Variable } & & $\begin{array}{l}\text { Logit Model }(n= \\
1,550)\end{array}$ & $\begin{array}{l}\text { GLM Model }(n= \\
952)\end{array}$ \\
\hline & & $\begin{array}{l}\text { Odds Ratio (95\% } \\
\mathrm{Cl} \S)\end{array}$ & $\begin{array}{l}\text { Coefficient }(95 \% \\
\mathrm{Cl} \$)\end{array}$ \\
\hline $\begin{array}{l}\text { Physical/Psychological } \\
\text { Disability } \\
\text { (Ref: No) }\end{array}$ & Yes & $1.41(0.92 \sim 2.17)$ & $\begin{array}{l}0.10(-0.19 \sim \\
0.40)\end{array}$ \\
\hline $\begin{array}{l}\text { Types of Medical } \\
\text { Institution } \\
\text { (Ref: General Hospital) }\end{array}$ & Tertiary hospital & $1.92^{\dagger}(1.47 \sim 2.51)$ & $\begin{array}{l}-0.01(-0.23 \sim \\
0.20)\end{array}$ \\
\hline
\end{tabular}

Note. ${ }^{\S} \mathrm{Cl}=$ Confidence Interval ${ }^{*} p<0.05+p<0.00$

Table 4 describes the incremental expenditures on caregiving costs of total study participants. Elderly inpatients spend more than nonelderly patients by approximately US\$290 (95\% Cl: US\$127 US\$ 453) on caregiving costs after controlling for predisposing factors, enabling factors, and needs factors. Additionally, those who received financial support for their healthcare spent on average US\$254 (95\% Cl: US\$ 145 US\$ 362) more than non-receivers. Similarly, inpatients diagnosed with a CCI score greater than 1 spent on average US\$ 249 (95\% Cl: US\$16 US\$ 482) more than inpatients with a CCI score of zero. In addition, inpatients who had completed junior high school or less spent an average of US\$151 (95\% Cl: US\$ 4 US\$ 299) more than individuals with more than a senior high school education.

Table 4. The incremental caregiving costs among elderly and nonelderly inpatients 


\begin{tabular}{|c|c|c|}
\hline \multicolumn{2}{|l|}{ Variable } & \multirow{2}{*}{$\begin{array}{l}\text { Incremental Cost (US\$, 95\% } \\
\text { CI\$) } \\
-75(-187 \sim 36)\end{array}$} \\
\hline $\begin{array}{l}\text { Gender } \\
\text { (Ref: Male) }\end{array}$ & Female & \\
\hline $\begin{array}{l}\text { Age Group } \\
\text { (Ref: Nonelderly group) }\end{array}$ & Elderly group ( $\geq 65$ ) & $290^{\dagger}(127 \sim 453)$ \\
\hline $\begin{array}{l}\text { Educational Level } \\
\text { (Ref: } \geq \text { Senior High) }\end{array}$ & $\leq$ Junior high & $151^{*}(4 \sim 299)$ \\
\hline \multirow{3}{*}{$\begin{array}{l}\text { Household Type } \\
\text { (Ref: With spouse) }\end{array}$} & Alone & $-111(-262 \sim 40)$ \\
\hline & $\begin{array}{l}\text { With spouse and another } \\
\text { member }\end{array}$ & $99(-74 \sim 273)$ \\
\hline & With another member & $73(-128 \sim 274)$ \\
\hline \multirow{4}{*}{$\begin{array}{l}\text { Household Income } \\
\text { (Ref: Low (1st)) }\end{array}$} & Low-Middle (2nd ) & $-89(-261 \sim 83)$ \\
\hline & Middle (3rd ) & $-54(-250 \sim 141)$ \\
\hline & Middle-High (4th ) & $-89(-28 \sim 106)$ \\
\hline & High (5th ) & $-47(-296 \sim 202)$ \\
\hline $\begin{array}{l}\text { Type of Public Insurance } \\
\text { (Ref: NHI) }\end{array}$ & Medicaid Type 1/Type 2 & $151(-117 \sim 420)$ \\
\hline $\begin{array}{l}\text { Financial Support } \\
\text { (Ref: Non-Receiver) }\end{array}$ & Receiver & $254^{\dagger}(145 \sim 362)$ \\
\hline $\begin{array}{l}\text { Number of Chronic diseases } \\
\text { (Ref: 1) }\end{array}$ & $\geq 2$ & $222(-42 \sim 488)$ \\
\hline $\begin{array}{l}\mathrm{CCl} \\
\text { (Ref: 0) }\end{array}$ & $\geq 1$ & $249^{*}(16 \sim 482)$ \\
\hline $\begin{array}{l}\text { Physical/Psychological } \\
\text { Disability } \\
\text { (Ref: No) }\end{array}$ & Yes & $106(-84 \sim 298)$ \\
\hline $\begin{array}{l}\text { Types of Medical Institution } \\
\text { (Ref: General Hospital) }\end{array}$ & Tertiary hospital & $108(-11 \sim 229)$ \\
\hline
\end{tabular}

\section{Note}


adjusted to US\$, $\S \mathrm{Cl}=$ Confidence Interval

${ }^{*} \mathrm{p}<0.05+p<0.00$

\section{Determinants of caregiver use and caregiving costs among elderly inpatients}

Table 5 demonstrates the results of two-part regression among elderly inpatients. Elderly inpatients who reside by themselves were less likely to use caregivers (OR: $0.48, p<0.00)$ than those who live with a spouse, but household type had no significant relationship with caregiving costs $(\beta: 0.01, p=0.97)$. However, those who had received financial support for healthcare management (OR: 2.70, $p<0.00$ ), had a physical or psychological disability (OR: 1.76, $\mathrm{p}<0.05$ ), or used the tertiary hospital for inpatient service (OR: 2.44, $p<0.00$ ) were more likely to use caregivers during hospitalization. Of these, patients with financial support $(\beta: 0.55, p<0.00)$ spent $55 \%$ more on caregiving costs than non-receivers, and patients diagnosed with more than two chronic diseases $(\beta$ : $0.66, p<0.05)$ spent $66 \%$ more on caregiving costs than patients with a single disease. Additionally, elderly patients who received financial support spent on average approximately US\$ 552 (95\% Cl: US\$328 US\$ 776) more on caregiving costs than nonreceivers (Table 6). Likewise, patients with more than two chronic diseases spent an average of US\$567 (95\% Cl: US\$ $106 \sim$ US\$1,029) more than patients with a single disease. 
Table 5

Analysis of caregiver use and its costs among elderly inpatients

\begin{tabular}{|c|c|c|c|}
\hline \multirow[t]{2}{*}{ Variable } & & \multirow{2}{*}{$\begin{array}{l}\text { Logit Model }(n= \\
819) \\
\text { Odds Ratio ( } 95 \% \\
\text { Cl\$) }\end{array}$} & \multirow{2}{*}{$\begin{array}{l}\text { GLM Model }(n= \\
574) \\
\text { Coefficient }(95 \% \\
\mathrm{Cl} \$)\end{array}$} \\
\hline & & & \\
\hline & \multirow[t]{2}{*}{ Female } & \multirow[t]{2}{*}{$\begin{array}{l}0.97(0.65 \sim \\
1.46)\end{array}$} & \multirow[t]{2}{*}{$\begin{array}{l}-0.05(-0.32 \sim \\
0.22)\end{array}$} \\
\hline (Ref: Male) & & & \\
\hline Educational Level & \multirow[t]{2}{*}{$\leq$ Junior High } & \multirow{2}{*}{$\begin{array}{l}0.73(0.44 \sim \\
1.21)\end{array}$} & \multirow{2}{*}{$\begin{array}{l}0.26(-0.06 \sim \\
0.59)\end{array}$} \\
\hline (Ref: $\geq$ Senior High) & & & \\
\hline \multirow{4}{*}{$\begin{array}{l}\text { Household Type } \\
\text { (Ref: With spouse) }\end{array}$} & \multirow[t]{2}{*}{ Alone } & \multirow{2}{*}{$\begin{array}{l}0.48^{\dagger} \\
0.75)\end{array}(0.30 \sim$} & \multirow{2}{*}{$\begin{array}{l}0.01(-0.32 \sim \\
0.33)\end{array}$} \\
\hline & & & \\
\hline & $\begin{array}{l}\text { With spouse and another } \\
\text { member }\end{array}$ & $\begin{array}{l}1.24(0.70 \sim \\
2.19)\end{array}$ & $\begin{array}{l}0.29(-0.08 \sim ~ \\
0.68)\end{array}$ \\
\hline & With another member & $\begin{array}{l}0.68(0.37 \sim \\
1.24)\end{array}$ & $\begin{array}{l}0.11(-0.27 \sim \\
0.50)\end{array}$ \\
\hline \multirow{4}{*}{$\begin{array}{l}\text { Household Income } \\
\text { (Ref: Low (1st )) }\end{array}$} & Low-Middle (2nd ) & $\begin{array}{l}0.92(0.59 \sim \\
1.42)\end{array}$ & $\begin{array}{l}0.01(-0.27 \sim \\
0.29)\end{array}$ \\
\hline & Middle (3rd ) & $\begin{array}{l}0.91(0.49 \sim \\
1.70)\end{array}$ & $\begin{array}{l}0.16(-0.25 \sim \\
0.58)\end{array}$ \\
\hline & Middle-High (4th ) & $\begin{array}{l}0.54(0.25 \sim \\
1.17)\end{array}$ & $\begin{array}{l}-0.14(-0.56 \sim \\
0.28)\end{array}$ \\
\hline & High (5th ) & $\begin{array}{l}0.89(0.41 \sim \\
1.92)\end{array}$ & $\begin{array}{l}0.28(-0.44 \sim \\
1.02)\end{array}$ \\
\hline $\begin{array}{l}\text { Type of Public Insurance } \\
\text { (Ref: NHI) }\end{array}$ & Medicaid Type 1/Type 2 & $\begin{array}{l}1.10(0.64 \sim \\
1.89)\end{array}$ & $\begin{array}{l}0.37(-0.06 \sim \\
0.82)\end{array}$ \\
\hline Financial Support & \multirow[t]{2}{*}{ Receiver } & \multirow{2}{*}{$\begin{array}{l}2.70^{\dagger}(1.84 \sim ~ \\
3.97)\end{array}$} & \multirow{2}{*}{$\begin{array}{l}0.55^{\dagger}(0.30 \sim \\
0.81)\end{array}$} \\
\hline (Ref: Non-Receiver) & & & \\
\hline $\begin{array}{l}\text { Number of Chronic } \\
\text { diseases }\end{array}$ & \multirow[t]{2}{*}{$\geq 2$} & \multirow[t]{2}{*}{$\begin{array}{l}1.03(0.62 \sim \\
1.70)\end{array}$} & \multirow[t]{2}{*}{$\begin{array}{l}0.66^{*} \\
1.05)\end{array}(0.28 \sim$} \\
\hline (Ref: 1) & & & \\
\hline $\mathrm{CCl}$ & \multirow[t]{2}{*}{$\geq 1$} & \multirow[t]{2}{*}{$\begin{array}{l}1.18(0.69 \sim \\
2.03)\end{array}$} & \multirow[t]{2}{*}{$\begin{array}{l}-0.14(-0.42 \sim \\
0.12)\end{array}$} \\
\hline (Ref: 0) & & & \\
\hline
\end{tabular}

Note. ${ }^{\S} \mathrm{Cl}=$ Confidence Interval ${ }^{*} \mathrm{p}<0.05+\mathrm{p}<0.00$ 


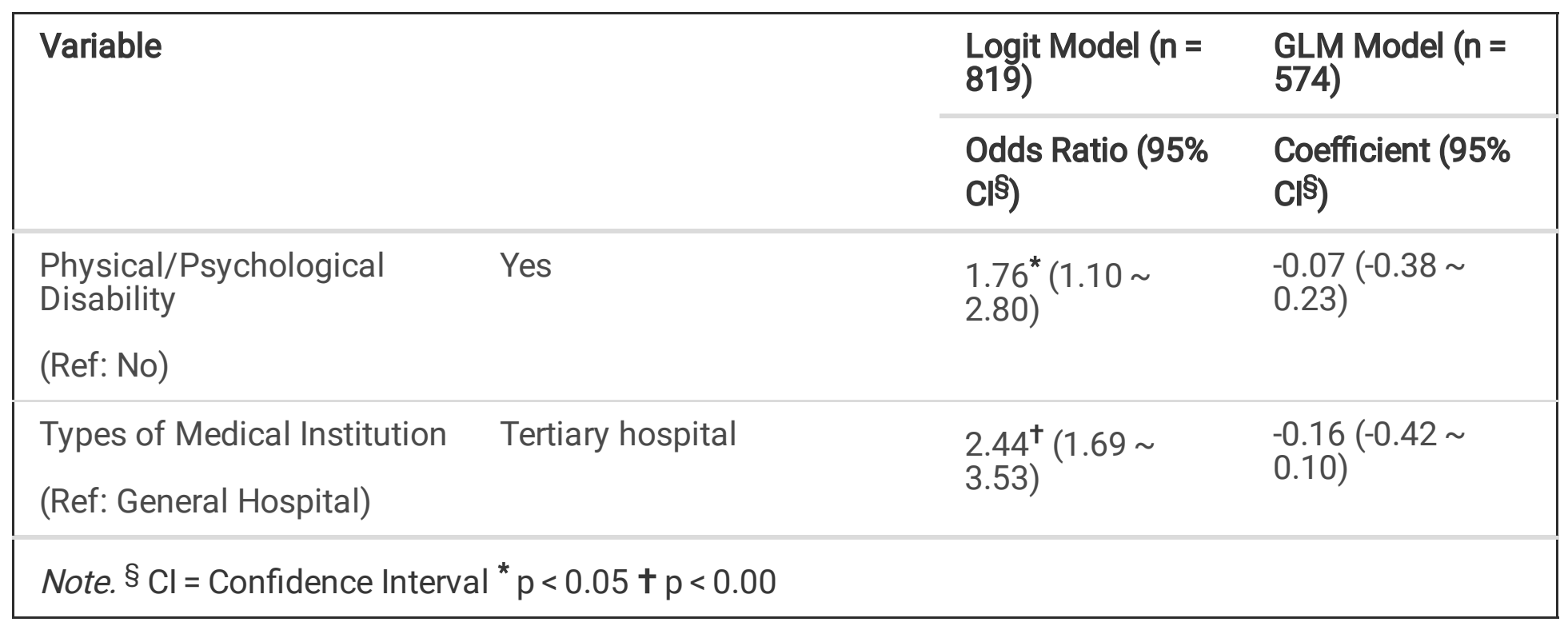

Table 6. The incremental caregiving costs among elderly inpatients 


\begin{tabular}{|c|c|c|}
\hline \multicolumn{2}{|l|}{ Variable } & \multirow{2}{*}{$\begin{array}{l}\text { Incremental Cost }(95 \% \mathrm{Cl} \text { ) } \\
-40(-247 \sim 167)\end{array}$} \\
\hline $\begin{array}{l}\text { Gender } \\
\text { (Ref: Male) }\end{array}$ & Female & \\
\hline $\begin{array}{l}\text { Educational Level } \\
\text { (Ref: } \geq \text { Senior High) }\end{array}$ & $\leq$ Junior High & $124(-97 \sim 347)$ \\
\hline \multirow{3}{*}{$\begin{array}{l}\text { Household Type } \\
\text { (Ref: With spouse) }\end{array}$} & Alone & $-129(-343 \sim 83)$ \\
\hline & With spouse and another member & $283(-94 \sim 661)$ \\
\hline & With another member & $7(-284 \sim 299)$ \\
\hline \multirow{4}{*}{$\begin{array}{l}\text { Household Income } \\
\text { (Ref: Low (1st)) }\end{array}$} & Low-Middle (2nd ) & $-6(-213 \sim 201)$ \\
\hline & Middle (3rd) & $102(-239 \sim 444)$ \\
\hline & Middle-High (4th ) & $-188(-454 \sim 77)$ \\
\hline & High (5th ) & $203(-463 \sim 870)$ \\
\hline $\begin{array}{l}\text { Type of Public Insurance } \\
\text { (Ref: } \mathrm{NHI} \text { ) }\end{array}$ & Medicaid Type 1/Type 2 & $331(-124 \sim 787)$ \\
\hline $\begin{array}{l}\text { Financial Support } \\
\text { (Ref: Non-Receiver) }\end{array}$ & Receiver & $552^{\dagger}(328 \sim 776)$ \\
\hline $\begin{array}{l}\text { Number of Chronic diseases } \\
\text { (Ref: 1) }\end{array}$ & $\geq 2$ & $567^{*}(106 \sim 1,029)$ \\
\hline $\begin{array}{l}\text { CCl } \\
\text { (Ref: 0) }\end{array}$ & $\geq 1$ & $-71(-276 \sim 133)$ \\
\hline $\begin{array}{l}\text { Physical/Psychological Disability } \\
\text { (Ref: No) }\end{array}$ & Yes & $43(-190 \sim 277)$ \\
\hline $\begin{array}{l}\text { Types of Medical Institution } \\
\text { (Ref: General Hospital) }\end{array}$ & Tertiary hospital & $63(-132 \sim 259)$ \\
\hline
\end{tabular}

\section{Note}

adjusted to US\$, $\S \mathrm{Cl}=$ Confidence Interval

${ }^{*} p<0.05+p<0.00$ 


\section{Determinants of caregiver use and caregiving costs among nonelderly inpatients}

Table 7 shows that nonelderly inpatients living alone (OR: $0.19, p<0.00)$ or residing with spouse and another family member (OR: $0.50, p<0.05)$ were less likely to use caregivers during hospital admission (Table 7). Additionally, those with low-middle income status were significantly less likely to be supported by caregivers than those with the lowest income status (OR: 025, $p<0.00$ ). However, similar to elderly inpatients, tertiary hospital users were more likely to receive assistance from caregivers (OR: 1.80, $p<$ $0.00)$. The analysis of the factors associated with caregiving costs among nonelderly inpatients shows that patients with financial support $(\beta: 0.26, p<0.00)$ or diagnosed with a $\mathrm{CCl}$ score greater than 1 ( $\beta$ : $0.80, p<0.00$ ) were associated with spending more on caregiving costs.

Additionally, the incremental caregiving costs among nonelderly inpatients showed that nonelderly inpatients with financial support spent on average US\$ 84 (95\% Cl: US\$ 7 US\$ 175) for caregiving costs (Table 8). In contrast to elderly inpatients, nonelderly inpatients with a $\mathrm{CCl}$ score greater than 1 spent on average US\$ 396 (95\% Cl: US\$ 60 US\$ 733) more on caregiving costs than those with CCl scores of zero. Although there was no significant relationship between gender and caregiving costs at the $95 \%$ significance level ( $\beta$ : 0.18, $p=0.08)$, nonelderly male inpatients spent an average of US\$ 93 (95\% Cl: US\$ $10 \sim$ US\$ 176) more on caregiving costs than nonelderly female inpatients. 
Table 7

Analysis of caregiver use and its costs among nonelderly inpatients

\begin{tabular}{|c|c|c|c|}
\hline \multirow[t]{2}{*}{ Variable } & & $\begin{array}{l}\text { Logit Model }(n= \\
731)\end{array}$ & $\begin{array}{l}\text { GLM Model }(n= \\
378)\end{array}$ \\
\hline & & $\begin{array}{l}\text { Odds Ratio (95\% } \\
\mathrm{Cl} \$)\end{array}$ & $\begin{array}{l}\text { Coefficient }(95 \% \\
\mathrm{Cl} \S)\end{array}$ \\
\hline $\begin{array}{l}\text { Gender } \\
\text { (Ref: Male) }\end{array}$ & Male & $\begin{array}{l}1.28(0.89 \sim \\
1.83)\end{array}$ & $\begin{array}{l}0.18(-0.02 \sim \\
0.39)\end{array}$ \\
\hline $\begin{array}{l}\text { Educational Level } \\
\text { (Ref: } \geq \text { Senior High) }\end{array}$ & $\leq$ Junior High & $\begin{array}{l}1.16(0.70 \sim \\
1.91)\end{array}$ & $\begin{array}{l}0.05(-0.21 \sim \\
0.33)\end{array}$ \\
\hline \multirow{3}{*}{$\begin{array}{l}\text { Household Type } \\
\text { (Ref: With spouse) }\end{array}$} & Alone & $\begin{array}{l}0.19^{\dagger} \\
0.56)\end{array}(0.06 \sim$ & $\begin{array}{l}-0.37(-1.08 \sim \\
0.34)\end{array}$ \\
\hline & $\begin{array}{l}\text { With spouse and another } \\
\text { member }\end{array}$ & $\begin{array}{l}0.50^{*}(0.30 \sim \\
0.85)\end{array}$ & $\begin{array}{l}0.94(-0.17 \sim \\
0.36)\end{array}$ \\
\hline & With another member & $\begin{array}{l}0.69(0.32 \sim \\
1.49)\end{array}$ & $\begin{array}{l}0.06(-0.37 \sim \\
0.50)\end{array}$ \\
\hline \multirow{4}{*}{$\begin{array}{l}\text { Household Income } \\
\text { (Ref: Low (1st)) }\end{array}$} & Low-Middle (2nd ) & $\begin{array}{l}0.25^{\dagger} \\
0.62)\end{array}(0.10 \sim$ & $\begin{array}{l}0.01(-0.62 \sim \\
0.65)\end{array}$ \\
\hline & Middle (3rd ) & $\begin{array}{l}0.64(0.26 \sim \\
1.58)\end{array}$ & $\begin{array}{l}-0.19(-0.80 \sim \\
0.41)\end{array}$ \\
\hline & Middle-High (4th ) & $\begin{array}{l}0.63(0.26 \sim \\
1.54)\end{array}$ & $\begin{array}{l}-0.27(-0.89 \sim \\
0.33)\end{array}$ \\
\hline & High (5th ) & $\begin{array}{l}0.64(0.26 \sim \\
1.55)\end{array}$ & $\begin{array}{l}-0.34(-0.97 \sim \\
0.27)\end{array}$ \\
\hline $\begin{array}{l}\text { Type of Public Insurance } \\
\text { (Ref: NHI) }\end{array}$ & Medicaid Type 1/Type 2 & $\begin{array}{l}0.69(0.23 \sim \\
2.02)\end{array}$ & $\begin{array}{l}0.55(-0.14 \sim \\
1.24)\end{array}$ \\
\hline $\begin{array}{l}\text { Financial Support } \\
\text { (Ref: Non-Receiver) }\end{array}$ & Receiver & $\begin{array}{l}1.02(0.71 \sim \\
1.46)\end{array}$ & $\begin{array}{l}0.26^{\dagger} \\
0.45)\end{array}(0.06 \sim$ \\
\hline $\begin{array}{l}\text { Number of Chronic } \\
\text { diseases } \\
\text { (Ref: 1) }\end{array}$ & $\geq 2$ & $\begin{array}{l}0.66(0.41 \sim \\
1.06)\end{array}$ & $\begin{array}{l}0.09(-0.18 \sim \\
0.37)\end{array}$ \\
\hline $\begin{array}{l}\text { CCl } \\
\text { (Ref: 0) }\end{array}$ & $\geq 1$ & $\begin{array}{l}1.33(0.77 \sim \\
2.31)\end{array}$ & $\begin{array}{l}0.80^{\dagger} \\
1.28)\end{array}(0.31 \sim$ \\
\hline
\end{tabular}

Note. ${ }^{\S} \mathrm{Cl}=$ Confidence Interval ${ }^{*} p<0.05+p<0.00$ 


\begin{tabular}{|c|c|c|c|}
\hline \multirow[t]{2}{*}{ Variable } & & \multirow{2}{*}{$\begin{array}{l}\text { Logit Model }(n= \\
\text { 731) } \\
\text { Odds Ratio (95\% } \\
\text { CI\$) }\end{array}$} & \multirow{2}{*}{$\begin{array}{l}\text { GLM Model }(n= \\
378) \\
\text { Coefficient }(95 \% \\
\mathrm{Cl} \$)\end{array}$} \\
\hline & & & \\
\hline $\begin{array}{l}\text { Physical/Psychological } \\
\text { Disability } \\
\text { (Ref: No) }\end{array}$ & Yes & $\begin{array}{l}1.29(0.56 \sim \\
2.91)\end{array}$ & $\begin{array}{l}0.32(-0.21 \sim \\
0.86)\end{array}$ \\
\hline $\begin{array}{l}\text { Types of Medical Institution } \\
\text { (Ref: General Hospital) }\end{array}$ & Tertiary hospital & $\begin{array}{l}1.80^{\dagger} \\
2.58)\end{array}(1.26 \sim$ & $\begin{array}{l}0.03(-0.20 \sim \\
0.27)\end{array}$ \\
\hline Note. $\S^{\mathrm{Cl}}=$ Confidence Interv & $p<0.05+p<0.00$ & & \\
\hline
\end{tabular}


Table 8

The incremental caregiving costs among nonelderly inpatients

\begin{tabular}{|c|c|c|}
\hline \multicolumn{2}{|l|}{ Variable } & \multirow{2}{*}{$\begin{array}{l}\text { Incremental Cost (95\% Cl\$) } \\
93^{*}(10 \sim 176)\end{array}$} \\
\hline $\begin{array}{l}\text { Gender } \\
\text { (Ref: Male) }\end{array}$ & Female & \\
\hline $\begin{array}{l}\text { Educational Level } \\
\text { (Ref: } \geq \text { Senior High) }\end{array}$ & $\leq$ Junior High & $39(-75 \sim 155)$ \\
\hline \multirow{3}{*}{$\begin{array}{l}\text { Household Type } \\
\text { (Ref: With spouse) }\end{array}$} & Alone & $-256^{\dagger}(-409 \sim-102)$ \\
\hline & With spouse and another member & $-55(-173 \sim 62)$ \\
\hline & With another member & $-23(-208 \sim 162)$ \\
\hline \multirow{4}{*}{$\begin{array}{l}\text { Household Income } \\
\text { (Ref: Low (1st )) }\end{array}$} & Low-Middle (2nd ) & $-217(-531 \sim 97)$ \\
\hline & Middle (3rd ) & $-142(-455 \sim 170)$ \\
\hline & Middle-High (4th ) & $-171(-484 \sim 141)$ \\
\hline & High (5th ) & $-191(-504 \sim 121)$ \\
\hline $\begin{array}{l}\text { Type of Public Insurance } \\
\text { (Ref: NHI) }\end{array}$ & Medicaid Type 1/Type 2 & $144(-245 \sim 534)$ \\
\hline $\begin{array}{l}\text { Financial Support } \\
\text { (Ref: Non-Receiver) }\end{array}$ & Receiver & $84^{*}(7 \sim 175)$ \\
\hline $\begin{array}{l}\text { Number of Chronic diseases } \\
\text { (Ref: 1) }\end{array}$ & $\geq 2$ & $-27(-135 \sim 81)$ \\
\hline $\begin{array}{l}\text { CCI } \\
\text { (Ref: 0) }\end{array}$ & $\geq 1$ & $396^{*}(60 \sim 733)$ \\
\hline $\begin{array}{l}\text { Physical/Psychological Disability } \\
\text { (Ref: No) }\end{array}$ & Yes & $163(-123 \sim 450)$ \\
\hline $\begin{array}{l}\text { Types of Medical Institution } \\
\text { (Ref: General Hospital) }\end{array}$ & Tertiary hospital & $91^{*}(8 \sim 175)$ \\
\hline
\end{tabular}

Note. adjusted to US\$, $\S \mathrm{Cl}=$ Confidence Interval

${ }^{*} p<0.05+p<0.00$

\section{Discussion}


This study demonstrated that elderly inpatients had almost twice a tendency to use caregivers and had more incremental caregiving costs than those of nonelderly inpatients. This study finds coincide points with previous studies $(9,19,26)$ that aging may put a substantial economic burden from caregiving costs, whereas figures noticeable aspects that the caregiver use and its costs distinctively vary according to the underlying health condition and access to social resources among elderly patients in Korea.

Elderly patients experiencing limited functions require 1.7 times more close-up support from caregivers than those without a disability. In the case of elderly patients with disability status, adequate interventions at the proper time for multiple dimensions, such as physical and psychological aspects, are continuously needed due to their high dependency on caretakers (27-29). In terms of traditional background in Korea, parental caregiving is a traditional norm that elderly patients have more tendency to rely on family members (30). Under this circumstance, family members of elderly patients are required to consume considerable time and take financial burden. Besides, elderly inpatients diagnosed with multiple chronic diseases are likely to spend $66 \%$ more on caregiving costs. This result supports previous studies showing that increased risk of morbidity is related to informal care network $(31,32)$. According to the results from Hong, elderly inpatients with higher medical needs are more likely to receive various caregiving services to cope with their daily routines (33). The underlying chronic physical and psychological health status would have deteriorated health restoration from acute illness and weighted the necessity of care during hospitalization. In contrast to the previous study (34), this study showed no significant relationship between the severity of chronic diseases and caregiving costs among elderly inpatients. It can be assumed that the focus of this study was on hospitalized patients with general grounds rather than focusing on patients with a particular type of major chronic disease. Therefore, morbidity status should be considered to be the priority consideration for caregiving expenditures among general chronically ill elderly patients.

Also, social capitals at both the individual and the aggregate level were related to caregiver use among elderly inpatients. Although both elderly and nonelderly patients shared some common factors related to caregiver use, elderly patients had a greater probability of using caregivers and spending more incremental caregiving costs. From the individual perspective, elderly patients who live by themselves had 0.48 times of lower chance of using caregivers during hospitalization. This finding supports Sok et al.'s study that seniors living alone had relatively fewer motives to engage in health-promoting behavior due to absence in the family living arrangement (35). It highlights that the elderly inpatients living alone have access to limited social support in their time of need, which may lead to a considerable risk of deteriorating health conditions. On the other hand, patients who receive benefits from private insurance or financial subsidies from nonprofit organizations are almost three times likely to use caregivers and having $55 \%$ times of spending caregiving costs. Following the study from Jeon et al., the probability of inpatient care utilization is in higher demand for health care among patients who hold private health insurance (36). Additionally, the review of the empirical studies showing the characteristics of private insurance holders under universal health care systems in Europe, Australia, and Israel presented that private insurance could be the influential factor for health care financing for those who can actually pay and have a higher tendency to receive more supports for managing health (37). It can be assumed that 
patients with a relatively lower financial burden have higher opportunity costs during inpatient care, and therefore have more incentives to use caregivers during hospitalization.

Similar to the previous context, inpatients at tertiary hospitals are 2.44 times more likely to use caregivers, showing that the influence of the healthcare system at the aggregate level could be a prime factor for deciding elderly inpatients' health behavior. This study classified the types of medical institutions according to which hospital patients had been used generally within the last year. This showed the general pattern of patients' selection of healthcare service utilization because the use of a major medical institution that has been generally used for hospitalization reflects patients' highest expectations for managing their health. Patients in Korea have more accessibility to using higher hospital-level, which shows a particular type of health behavior manifested in Korea as a high concentration of patients in large hospitals $(38,39)$. Based on this social aspect, patients with chronic conditions and acutely need to receive medical services are likely to use hospitals with highly equipped infrastructure with intensive care, which is a priority for their health management plans. Along with this trend, patients' tendency to use caregivers would be derived from the desire to utilize assured resources for managing their health.

While the present findings imply some significant social determinants associated with caregiver use and its expenses, they are not without several limitations. First, the functional ability level was not included in the analysis due to limited information. Although physical or psychological disability status was analyzed and exhibited a significant relationship with caregiver use, the degree of its association may differ by the magnitude of functional ability. Another limitation is that this study focused on inpatients with various types of diseases rather than considering patients with a particular disease. As a result, cases of critical health conditions such as terminal cancer are underrepresented. Therefore, this study may not adequately reflect the specific situations of those with severe health conditions. If the duration of the disease diagnosis or the treatment process required for a particular type of disease were given, patients' health status would be more precisely clarified. Last, there is a potential for recall bias that may cause the underestimating of the costs of informal caregiving since this study used self-reported data. However, this study used data on health utilization for inpatient services that were collected during a year, which is relatively short and may enhance the accuracy of the calculated healthcare costs. Regardless of these limitations, this study indicates the importance of identifying the caregiving cases of elderly inpatients and related expenditures while monitoring elderly patients' relapse cases and their social resources, which are the focal points to relieve the caregiving burden of elderly patients' households.

\section{Conclusion}

The use of caregivers and its costs are associated with elderly patients' underlying health conditions and their social capitals under the acute situation. The influences of health status and social determinants are differed by age groups, and therefore differential approaches to ease time and financial burdens should be given considering the aging effect. Sustainable healthcare management programs that periodically examine the prevalence of morbidity and assure the quality and assessment of social capital should be projected. 


\section{Declarations}

\section{Ethics approval and consent to participate}

Not applicable

\section{Consent for publication}

Not applicable

\section{Availability of data and materials}

The datasets used and analyzed during the current study are available in the Korea Health Panel repository, https://www.khp.re.kr:444/web/data/data.do

\section{Competing interests}

The authors declare that they have no competing interests with respect to the research, authorship, and/or publication of this article.

\section{Funding}

The authors declare there are no funding for this study.

\section{Author's contribution}

JK acquired a secondary dataset, analyzed, and interpreted the study results. SK contributed to study design and advised interpreting the results of the analysis. All authors reviewed the content of this study and approved the final manuscript.

\section{Acknowledgements}

study used Korea Health Panel Survey Data from 2017 which is jointly carried out by the Korea Institute for Health and Social Affairs and the National Health Insurance Service.

\section{Abbreviations}

CNS: Comprehensive Nursing Service

KHP: Korea Health Panel Study Data

LTIC: Long-term Care Insurance

NHI: National Health Insurance

CCl: Charlson Comorbidity Index 
KCD-7: The Korean Standard Classification of Disease Version-7

ICD-10: The International Statistical Classification of Disease and Related Health Problems

GLM: Generalized Linear Model

\section{References}

1. Statistics Research Institute SK. Future population estimates: Year 2017-2067 2019.

2. National Health Insurance Service HIRaASoK. National Health Insurance Statistical Yearbook: 2018. 2019.

3. Statistics Research Institute SK. Korean Social Trends 2019. 2019.

4. Kim JH, Kim S, Pask E, Jeong S, Lee E. Policy Issues and New Direction for Comprehensive Nursing Service in the National Health Insurance. Journal of Korean academy of nursing administration. 2017;23(3):312-22.

5. Kim BH, Kang HY. Job satisfaction, Job stress, Burnout, and Turnover Intention of Comprehensive Nursing Care Service Ward Nurses and General Ward Nurses. Journal of the Korea AcademiaIndustrial cooperation Soceity. 2018;19(5):459-69.

6. Souwer E, Bastiaannet E, de Bruijn S, Breugom A, van den Bos F, Portielje J, et al. Comprehensive multidisciplinary care program for elderly colorectal cancer patients:"From prehabilitation to independence". European Journal of Surgical Oncology. 2018;44(12):1894-900.

7. Lawrence VA, Hazuda HP, Cornell JE, Pederson T, Bradshaw PT, Mulrow CD, et al. Functional independence after major abdominal surgery in the elderly. Journal of the American College of Surgeons. 2004;199(5):762-72.

8. Richman IB, Brodie M. A national study of burdensome health care costs among non-elderly Americans. BMC health services research. 2014;14(1):1-7.

9. Tomita S, Hoshino E, Kamiya K, Yasuhiro O, Rahman M. Direct and indirect costs of home healthcare in Japan: A cross-sectional study. Health \& Social Care in the Community. 2020;28(3):1109-17.

10. Angioli R, Capriglione S, Aloisi A, Miranda A, de Cicco Nardone C, Terranova C, et al. Economic impact among family caregivers of patients with advanced ovarian cancer. International Journal of Gynecologic Cancer. 2015;25(8).

11. Joo H, Dunet DO, Fang J, Wang G. Cost of informal caregiving associated with stroke among the elderly in the United States. Neurology. 2014;83(20):1831-7.

12. Kim HB, Lim W. Long-term care insurance, informal care, and medical expenditures. Journal of Public Economics. 2015;125:128-42.

13. Kim HK, Kim SK, Shim HJ, Lee HM, Rhee H. A Convergence Study on Influencing Factors of Paid Care Service: Andersen's Behavioral Model. Journal of Digital Convergence. 2017;15(4):327-37.

14. Cho SH, Kim HR. Family and paid caregivers of hospitalized patients in Korea. Journal of clinical nursing. 2006;15(8):946-53. 
15. Kim Y, Jung Y, Kim JM, Lee T, Bae E, Song H, et al. Issues for Costing Method of Caregiving in Healthcare. Journal of Health Technology Assessment. 2014;2(2):93-8.

16. Lee T, Shin S, Song H, Park J, Jung Y, Bae E, et al. Costing Method of Caregiving in Healthcare. National Evidence-based Healthcare Collaborationg Agency. 2011;1(1):1-311.

17. Shin JW, Cho BH, Choi B, Shin J, Chun M, Lee E. 2018 Healthcare Experience Survey Ministry of Health and Welfare; 2018.

18. Andersen RM. Revisiting the behavioral model and access to medical care: does it matter? Journal of health and social behavior. 1995:1-10.

19. Heider D, Matschinger H, Müller H, Saum K-U, Quinzler R, Haefeli WE, et al. Health care costs in the elderly in Germany: an analysis applying Andersen's behavioral model of health care utilization. BMC health services research. 2014;14(1):71.

20. de Boer AG, Wijker W, de Haes HC. Predictors of health care utilization in the chronically ill: a review of the literature. Health Policy. 1997;42(2):101-15.

21. Jiang M, Yang G, Fang L, Wan J, Yang Y, Wang Y. Factors associated with healthcare utilization among community-dwelling elderly in Shanghai, China. PloS one. 2018;13(12):e0207646.

22. Bähler $C$, Huber CA, Brüngger B, Reich O. Multimorbidity, health care utilization and costs in an elderly community-dwelling population: a claims data based observational study. BMC health services research. 2015;15(1):23.

23. Kim K, Shin Y. The effect of the Policy of Expanding Coverage for Four major Diseases: Focused on Out-of-Pocket Payment. Health and Social Welfare Review. 2017;37(2):452-76.

24. Lee $Y$, Kim H, Jeong H, Noh Y. Patterns of Multimorbidity in Adults: An Association Rules Analysis Using the Korea Health Panel. International Journal of Environmental Research and Public Health. 2020;17(8):2618.

25. Kang C, Lee JH, Kim DK, Lee C, Oh JH. Current Status of Having a Usual Source of Care and Its Associated Factors in Korean Adults with Hypertension. Korean Journal of Family Practice. 2018;8(4):607-15.

26. Hayman JA, Langa KM, Kabeto MU, Katz SJ, DeMonner SM, Chernew ME, et al. Estimating the cost of informal caregiving for elderly patients with cancer. Journal of Clinical Oncology. 2001;19(13):3219-25.

27. Hilton JM, Kopera-Frye K, Krave A. Successful aging from the perspective of family caregivers. The Family Journal. 2009;17(1):39-50.

28. Sim SS, Lee DS. A Study on Satisfaction with Care Service depending on Activities of Daily Living and Type of Caregivers among Inpatients with Chronic Disease. The Korean Journal of Rehabilitation Nursing. 2012;15(1):11-9.

29. Rezende TCB, Coimbra AMV, Costallat LTL, Coimbra IB. Factors of high impacts on the life of caregivers of disabled elderly. Archives of Gerontology and Geriatrics. 2010;51(1):76-80. 
30. Kim JS, Lee EH. Cultural and noncultural predictors of health outcomes in Korean daughter and daughter-in-law caregivers. Public Health Nursing. 2003;20(2):111-9.

31. Andersson MA, Monin JK. Informal care networks in the context of multimorbidity: size, composition, and associations with recipient psychological well-being. Journal of aging and health. 2018;30(4):641-64.

32. Amer Nordin A, Mohd Hairi F, Choo WY, Hairi NN. Care Recipient Multimorbidity and Health Impacts on Informal Caregivers: A Systematic Review. The Gerontologist. 2019;59(5):e611-e28.

33. Hong SI. Understanding patterns of service utilization among informal caregivers of community older adults. The Gerontologist. 2010;50(1):87-99.

34. Li YN, Nong Dx, Wei B, Feng QM, Luo H. The impact of predisposing, enabling, and need factors in utilization of health services among rural residents in Guangxi, China. BMC health services research. 2016;16(1):1-9.

35. Sok SR, Yun EK. A comparison of physical health status, self-esteem, family support and healthpromoting behaviours between aged living alone and living with family in Korea. Journal of Clinical Nursing. 2011;20(11-12):1606-12.

36. Jeon B, Kwon S. Effect of private health insurance on health care utilization in a universal public insurance system: a case of South Korea. Health policy. 2013;113(1-2):69-76.

37. Kiil A. What characterises the privately insured in universal health care systems? A review of the empirical evidence. Health Policy. 2012;106(1):60-75.

38. Kang H. Policy Direction for Decreasing the Concentration of Patients to Extra-large Hospitals. Health Welfare Policy Forum. 2014;2014(4).

39. Jang SI. The improvement strategy and causes of the patients' concentrations on large hospitals. Public Health Affairs. 2019;3(1):165-9.

\section{Figures}




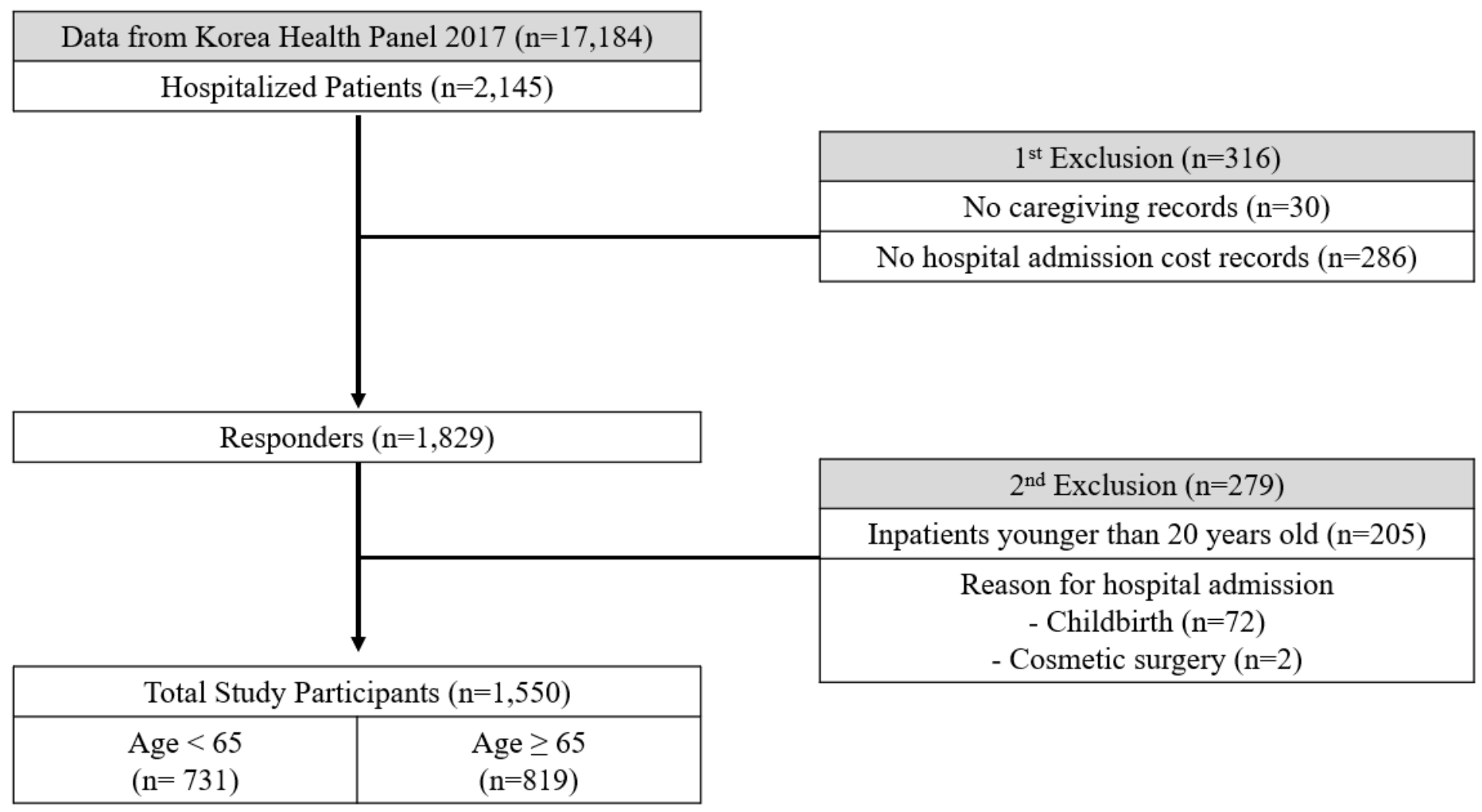

\section{Figure 1}

Flow diagram for the selection of study participants

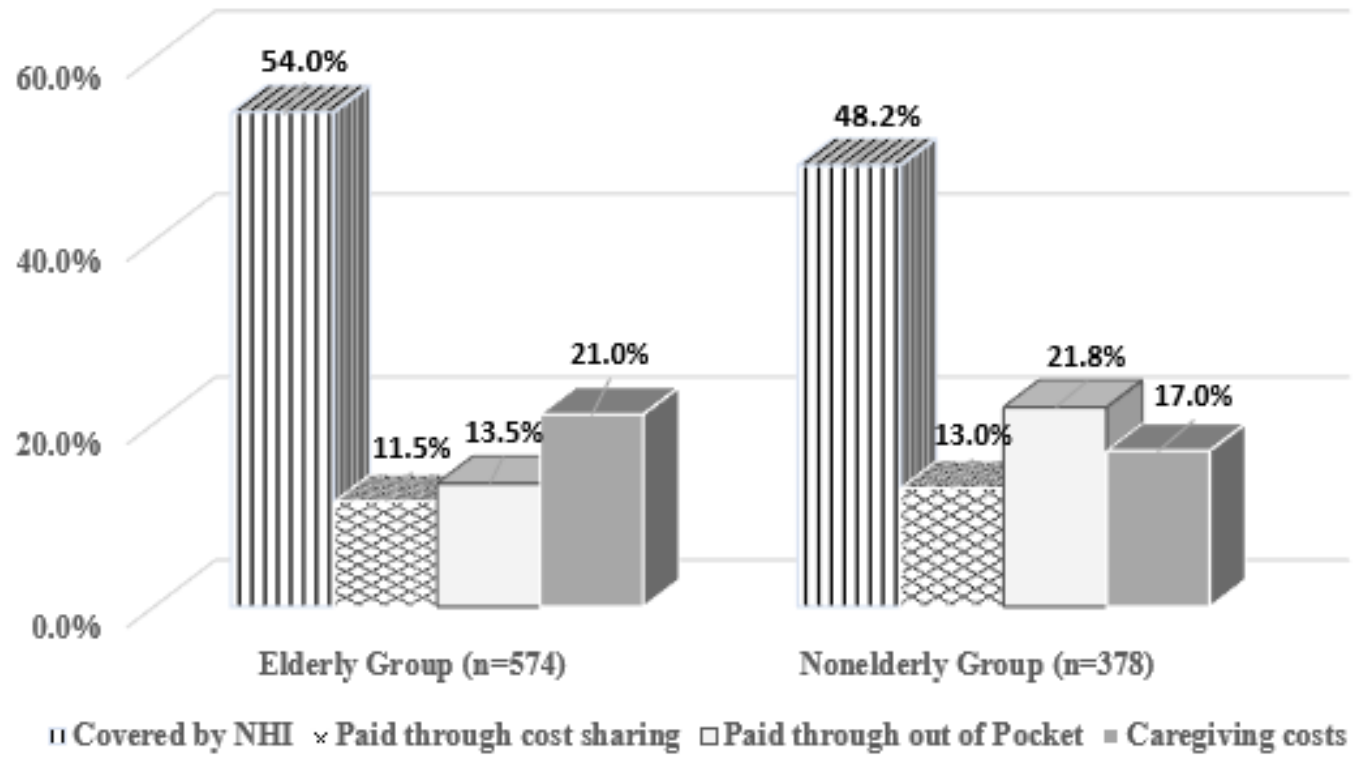

\section{Figure 2}

Direct Healthcare Expenditures and Caregiving Costs share on Inpatient Service 